\title{
Cytogenetic and molecular cytogenetic findings in giant dedifferentiated liposarcoma of the thigh
}

\author{
JUN NISHIO $^{1}$, MIKIKO AOKI ${ }^{2}$, KAZUKI NABESHIMA $^{2}$, HIROSHI IWASAKI $^{2}$ and MASATOSHI NAITO ${ }^{1}$ \\ Departments of ${ }^{1}$ Orthopaedic Surgery, ${ }^{2}$ Pathology, Faculty of Medicine, Fukuoka University, Fukuoka, Japan
}

Received August 30, 2011; Accepted November 4, 2011

DOI: $10.3892 /$ or.2011.1584

\begin{abstract}
Non-retroperitoneal dedifferentiated liposarcoma (DDLS) is relatively uncommon and its characterization at the molecular genetic level has been limited. We describe the cytogenetic and molecular cytogenetic findings of giant DDLS arising in the right thigh of an 83-year-old woman. Magnetic resonance imaging revealed a mass composed of two components with heterogeneous signal intensities, suggesting the coexistence of a fatty area and another soft tissue component. A wide resection of the tumor was performed. The resected, grossly heterogeneous mass, measuring 26x18x8 cm, was histopathologically composed of a well-differentiated liposarcomatous component transitioning abruptly into a dedifferentiated one. Cytogenetic analysis exhibited a complex karyotype with several numerical and structural alterations, including ring and giant marker chromosomes. Metaphase-based comparative genomic hybridization analysis showed high-level amplifications of 1q21-q25 and 12q13-q21. Interphase fluorescence in situ hybridization analysis revealed $M D M 2$ and $C D K 4$ gene amplification in both the well-differentiated and dedifferentiated components. These findings indicate that DDLS of the extremity shares a similar genetic background to retroperitoneal DDLS.
\end{abstract}

\section{Introduction}

Dedifferentiated liposarcoma (DDLS) is a distinct subtype of liposarcoma, showing transition from well-differentiated liposarcoma (WDLS) to non-lipogenic sarcoma of variable histologic grade. It occurs typically in the retroperitoneum of elderly individuals with no gender predilection. The phenomenon of dedifferentiation in DDLS is essentially considered to be time-dependent. DDLS has a high local recurrence rate but lower metastatic potential than other high-grade sarcomas (1). Due to the histologic complexity of DDLS, many differential diagnoses may be raised on the morphologic aspect alone.

Correspondence to: Dr Jun Nishio, Department of Orthopaedic Surgery, Faculty of Medicine, Fukuoka University, 7-45-1 Nanakuma, Jonan-ku, Fukuoka 814-0180, Japan

E-mail: jnishio@cis.fukuoka-u.ac.jp

Key words: dedifferentiated liposarcoma, cytogenetics, molecular genetics
Cytogenetically, DDLS is characterized by the presence of supernumerary ring and/or giant marker chromosomes $(2,3)$. Fluorescence in situ hybridization (FISH) and comparative genomic hybridization (CGH) studies have demonstrated that ring and giant marker chromosomes are composed, exclusively or partly, of amplified 12q13-q15 material, involving MDM2 and CDK4 (4-6). We also showed that giant marker chromosomes were composed partly of chromosome 12 material in a human cell line established from a retroperitoneal DDLS (7).

In contrast to retroperitoneal DDLS, cytogenetic and molecular biological analyses of non-retroperitoneal DDLS are few (1-3). We describe the cytogenetic and molecular cytogenetic findings of giant DDLS arising in the thigh of an elderly female.

\section{Materials and methods}

Case presentation. An 83-year-old Japanese woman was referred to our hospital with a history of a non-painful right thigh mass first noticed 2 months previously. Physical examination revealed an $18 \times 10 \mathrm{~cm}$, firm, non-tender mass in the posteromedial aspect of the right thigh. Magnetic resonance imaging clearly revealed that the mass was composed of two components. One was homogeneously low signal intensity on T1-weighted sequences with heterogeneously high signal intensity on T2-weighted spectral presaturation with inversion recovery (SPIR) sequences. The other was high signal intensity on T1-weighted sequences with low signal intensity on T2-weighted SPIR sequences, suggesting a lipomatous component (Fig. 1A and B). Contrast-enhanced T1-weighted sequences showed heterogeneous enhancement of the mass (Fig. 1C). Therefore, DDLS or mixed-type liposarcoma was suspected. She underwent an open biopsy and the pathologic diagnosis of WDLS was made. A wide resection of the tumor was performed. Macroscopically, the resected tumor, measuring $26 \times 18 \times 8 \mathrm{~cm}$, was composed of two portions with distinct colors, i.e., yellow and tan-gray. Microscopically, the tumor exhibited biphasic morphology consistent with the macroscopic appearance (Fig. 2A). The yellow component was composed of a proliferation of mature adipocytic cells with hyperchromtic nuclei and multivacuolated lipoblasts, representing WDLS (Fig. 2B). The tan-gray component was composed of a mixture of atypical spindle cells, round cells, and bizarre giant cells, resembling pleomorphic malignant fibrous histiocytoma (MFH) (Fig. 2C). The final pathologic diagnosis was DDLS. At 15 months of follow-up, the patient is alive without any evidence of local recurrence or distant metastasis. 

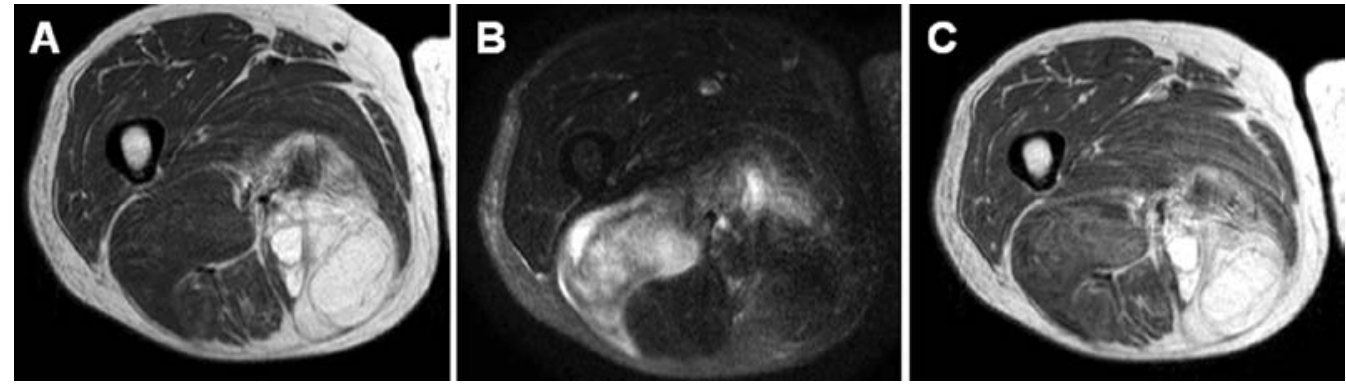

Figure 1. Axial magnetic resonance images of dedifferentiated liposarcoma involving the right thigh. The mass is composed of two components. (A) T1-weighted sequence shows that the medial component has high signal intensity and the lateral component has homogeneously low signal intensity. (B) T2-weighted spectral presaturation with inversion recovery sequence shows that the medial component has low signal intensity and the lateral component has heterogeneously high signal intensity. (C) Contrast-enhanced T1-weighted sequence reveals heterogenous enhancement throughout the mass.

Cytogenetic analysis. Representative fresh tissues were obtained from the surgical resection. Culturing, harvesting, and preparation of slides were performed as described previously (8). Cytogenetic analysis was performed on GTG-banded (Giemsa/ trypsin) metaphases, and karyotypic descriptions were expressed according to the International System for Human Cytogenetic Nomenclature 2009 (9).

Molecular cytogenetic analysis. CGH was performed as described previously (10). Briefly, DNA from the fresh frozen tissue was directly labeled with fluorescein-12-dUTP (Roche Diagnostics, Mannheim, Germany) by nick translation, with the use of a commercial kit (Abbott Molecular, Des Plaines, IL, USA). As a normal reference DNA, we used the Spectrum Red directed-labeled male total human DNA (Abbott Molecular). Subsequently, equal amounts of normal and tumor labeled probes $(800 \mathrm{ng}$ ) and $20 \mu \mathrm{g}$ of Cot-1 DNA (Gibco/BRL, Gaithersburg, MD, USA) were coprecipitated with ethanol. The precipitated DNA was dissolved in $10 \mu \mathrm{l}$ of hybridization buffer and denatured at $75^{\circ} \mathrm{C}$ for $8 \mathrm{~min}$. Normal metaphase spreads (Abbott Molecular) were denatured for $3 \mathrm{~min}$ at $75^{\circ} \mathrm{C}$ and hybridized with the DNA mixture in a moist chamber for 2 days. Slides were washed according to the protocol supplied by the manufacturer. Chromosomes were counterstained with 4,6-diamino-2-phenylindole (DAPI; Sigma, St. Louis, MO, USA). The location of aberrant CGH signals was analyzed using an image analysis system (Isis, Carl Zeiss Vision, Oberkochen, Germany) based on an integrated high-sensitivity monochrome charge-coupled device camera and automated CGH analysis software (MetaSystems GmbH, Altlussheim, Germany). Threecolor images, green (fluorescein-12-dUTP) for the tumor DNA, red (Spectrum Red) for the reference DNA, and blue (DAPI) for the DNA counterstain, were acquired from at least 10 metaphases. Only metaphases of good quality with strong, uniform hybridization were included in the analysis. Based on the control experiments, 1.2 and 0.8 were used as cutoff levels for gains and losses, respectively. Gains exceeding the 1.5 threshold were termed high-level amplifications. The heterochromatic regions in chromosomes 1,9, and 16, the p-arms of the acrocentric chromosomes, and Y chromosome were excluded from the analysis because of suppression of hybridization with Cot-1 DNA in these regions.

FISH was performed on paraffin-embedded tissue sections utilizing a commercially available Poseidon ${ }^{\mathrm{TM}}$ Repeat-Free $^{\mathrm{TM}}$
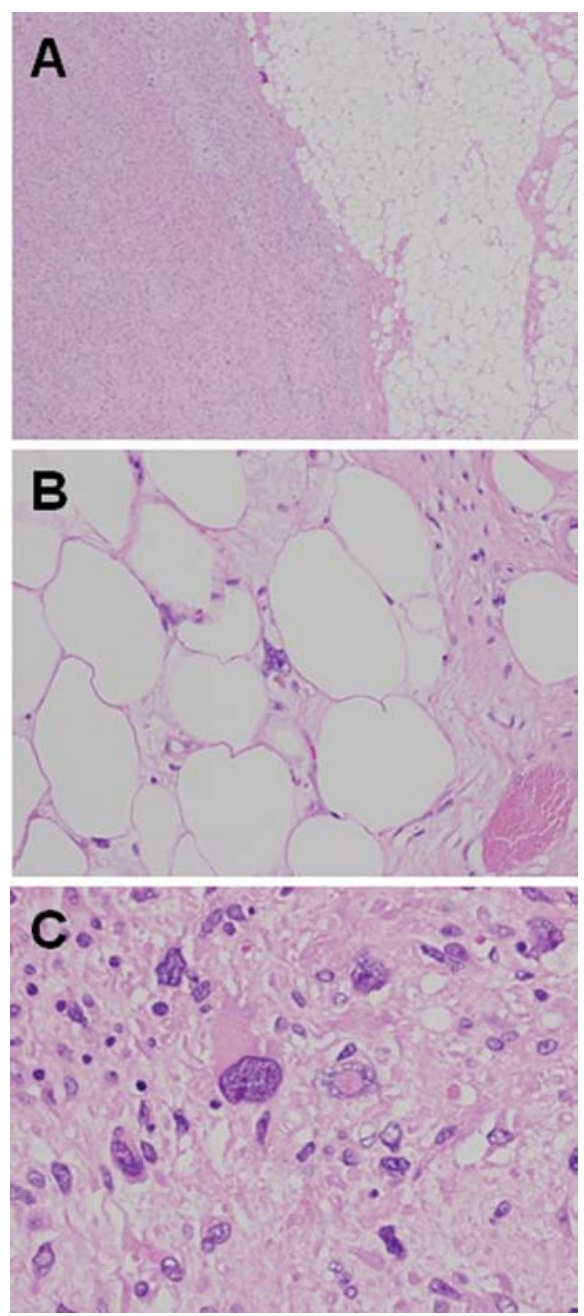

Figure 2. Microscopic findings of dedifferentiated liposarcoma. (A) Abrupt transition between well-differentiated liposarcoma and non-lipogenic area is seen. (B) The yellow component is composed of a proliferation of mature adipocytic cells with hyperchromtic nuclei, representing well-differentiated liposarcoma. (C) The tan-gray component is composed of a mixture of atypical spindle cells, round cells, and bizarre giant cells, resembling pleomorphic malignant fibrous histiocytoma.

MDM2 (12q15) or CDK4 (12q13)/SE12 control probe (Kreatech Diagnostics, Amsterdam, The Netherlands). Briefly, 4- $\mu \mathrm{m}$-thick paraffin-embedded tissue sections were deparaffinized, dehydrated, and incubated with pepsin according to 


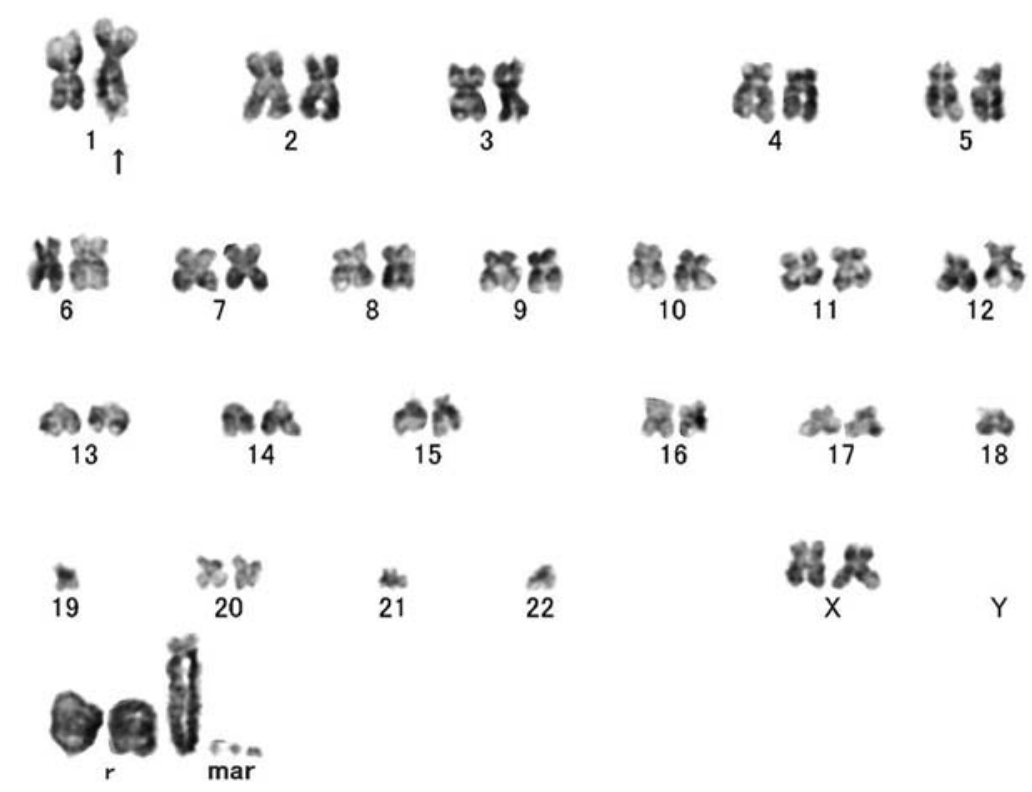

Figure 3. A representative GTG-banded karyotype of dedifferentiated liposarcoma, including ring and giant marker chromosomes. An arrow indicates the structural chromosome aberration.
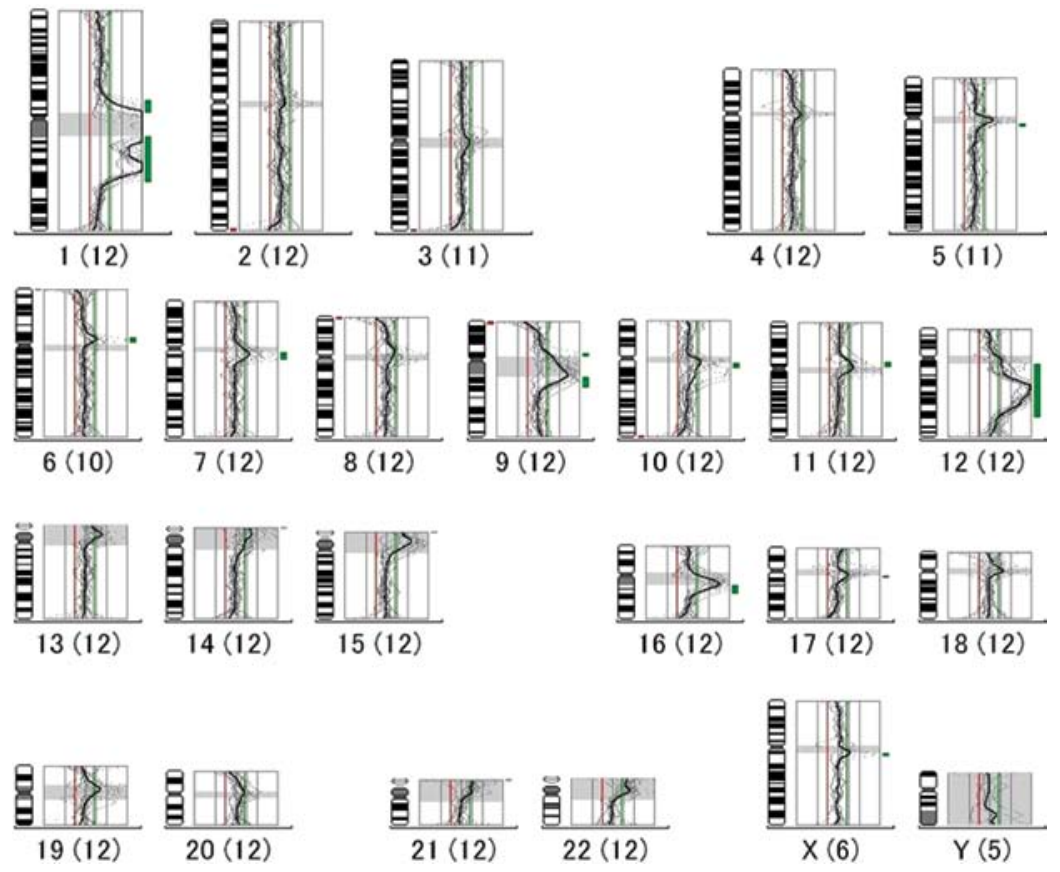

Figure 4. Comparative genomic hybridization profile of dedifferentiated liposarcoma showing high-level amplifications of 1q21-q25 and 12q13-q21. The line in the middle (gray) is the baseline ratio (1.0); the left (red) and right (green) lines indicate ratio values of 0.8 and 1.2, respectively. Bars on the left (red) and right (green) of each frame indicate losses and gains, respectively. The terminology 1 (12) represents 12 aberrations detected on chromosome 1 . The same applies to other chromosomes shown in the profile.

the manufacturer's instructions. The probe and slides were co-denatured at $80^{\circ} \mathrm{C}$ for $5 \mathrm{~min}$ and incubated at $37^{\circ} \mathrm{C}$ overnight in a humidified chamber. Post-hybridization washing was performed following standard procedures. The slides were counterstained with DAPI. A minimum of 100 interphase nuclei with strong, well-delineated signals and distinct nuclear borders were scrutinized. An $M D M 2$ or $C D K 4 / \mathrm{SE} 12$ ratio of $\geq 2.0$ was considered amplified for the $M D M 2$ or $C D K 4$ gene, whereas an $M D M 2$ or $C D K 4 / \mathrm{SE} 12$ ratio of $<2.0$ was considered non-amplified.

\section{Results}

Cytogenetic analysis revealed a complex karyotype with several numerical and structural alterations, including ring and giant marker chromosomes (Fig. 3). The composite karyotype was as follows: 44-48,XX,dic(1;?)(q32;?),-13,-18,-19,-21,-22,+1$2 r,+1-4 \operatorname{mar}[\mathrm{cp} 11]$.

Metaphase-based CGH analysis showed high-level amplifications of 1q21-q25 and 12q13-q21. Significant loss of DNA sequences was not found. This CGH profile is shown in Fig. 4. 

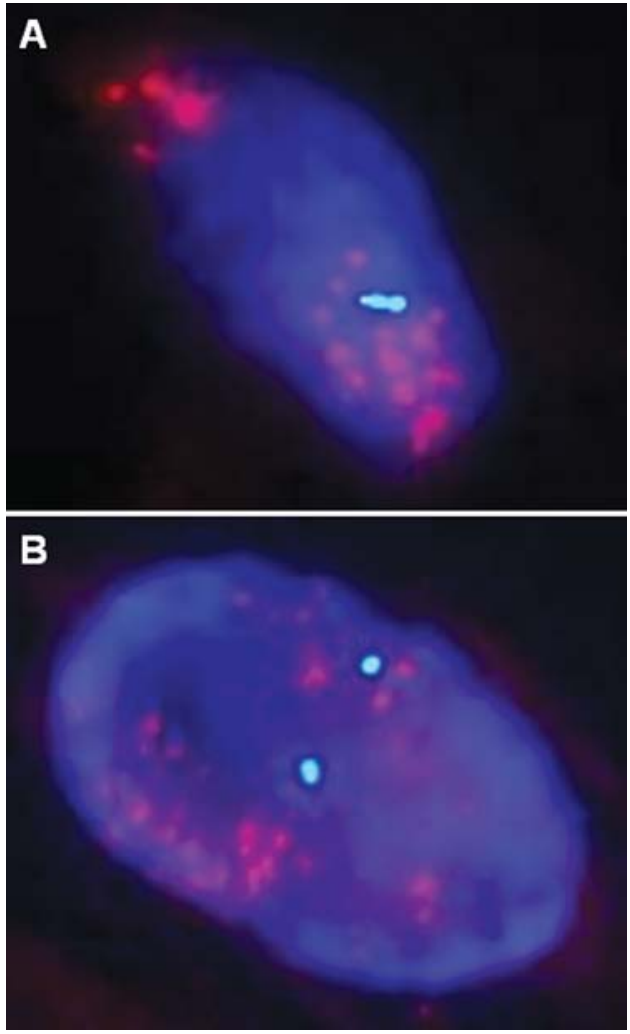

Figure 5. Fluorescence in situ hybridization with $C D K 4$ (red signal)/SE12 (green signal) on paraffin-embedded sections from the well-differentiated liposarcomatous component (A) and the dedifferentiated component (B). High-level amplification of $C D K 4$ is observed in the cell nuclei.

Interphase FISH analysis showed high-level amplification ( $>10$ copies per cell) of the MDM2 and $C D K 4$ genes in both the well-differentiated and dedifferentiated components (Fig. 5).

\section{Discussion}

Dedifferentiation occurs most frequently in retroperitoneal liposarcomas (11) and can manifest a variety of histologic patterns, resembling pleomorphic $\mathrm{MFH}$, high-grade myxofibrosarcoma, or adult-type fibrosarcoma. The concept of low-grade dedifferentiation has increasingly been recognized (11). A peculiar neural-like or meningothelial-like whirling pattern has also been reported (12-14). This pattern is often associated with metaplastic bone formation. Interestingly, several authors suggested that this unusual type of DDLS shares many of the cytogenetic and molecular genetic features with more typical DDLS $(14,15)$.

Similar to WDLS, DDLS is characterized by the presence of supernumerary ring and/or giant marker chromosomes containing amplified genomic sequences in the $12 \mathrm{q} 13-\mathrm{q} 15$ region. This amplification includes approximately 150 genes, including several of major potential relevance such as $M D M 2$, $C D K 4, H M G A 2$, and TSPAN31 (formerly known as SAS) (16-18). $M D M 2$ is essential for ubiquitnation and degradation of the tumor suppressor p53. MDM2 amplification is, therefore, thought to result in reduced levels of p53. CDK4 encodes a $33-\mathrm{kD}$ protein that is a key factor in the regulation of the G1-S translation of the cell cycle. Accumulation of the CDK4-CCDN1 complex leads to phosphorylation of the RB1 protein. It has been proposed that FISH analysis for MDM2 and $C D K 4$ amplification is a valuable adjunct in the diagnosis of DDLS (19), especially when the transition from WDLS to DDLS cannot be observed by histology. The cytogenetic and molecular cytogenetic findings of the present case showed the characteristic features of DDLS.

In addition to the 12q13-q15 amplification, high-level amplifications of 1p32 and 6q23 have been identified in DDLS $(5,6,17)$. It is of great interest that co-amplifications of $1 \mathrm{p} 32$ and 6q23 have never been observed in WDLS. JUN and ASK1 are up-regulated through amplifications in 1p32 and 6q23, respectively $(20,21)$. $J U N$ encodes part of the activator protein transcription factor (AP-1) complex involved in cell proliferation, transformation, and apoptosis and inhibits peroxisome proliferator-activated receptor (PPAR) $-\gamma$ through $\mathrm{C} / \mathrm{EBP}$ beta $(20,22)$. ASK1 encodes a MAP3 kinase involved in the Jun N-terminal kinase (JNK) signaling pathway (21). ASK1 amplification activates JNK ultimately leading to JUN activation and PPAR $-\gamma$ inactivation. It has been shown that PPAR- $\gamma$ plays a key role in adipocytic differentiation (23). Therefore, amplification of $J U N$ and $A S K 1$ may directly block adipocytic differentiation in DDLS. On the other hand, we were not able to detect any gain or amplification in 1p32 and 6q23. Most recently, Tap et al (6) reported that $6 \mathrm{q}$ amplification occurred primarily in retroperitoneal DDLS. The clinical and biological significance of this observation needs to be evaluated in a large series of DDLS.

High-level amplification of 1q21-q25 was also found in the present case. Extra copies of sequences from this region have been described to occur frequently in soft tissue sarcomas, including DDLS (5,24-26). Nilsson et al (27) reported that the COAS1-3 genes, located at 1q21, were found to be amplified at different levels in adipocytic tumors. The most common localization of extra COAS signals in adipocytic tumors was in ring and giant marker chromosomes. Kresse et al (28) suggested that ATF6 and DUSP12 may be the most likely candidate target genes for the 1q23 amplification in liposarcomas. Of note, Forus et al (4) suggested that 1q amplification may be indicative of a more malignant phenotype and ability of metastasis in WDLS.

More recently, Hélias-Rodzewicz et al (29) reported amplification of either 3p12 or 11q22 chromosomal bands in a subset of DDLS. VGLL3 and YAP1 are up-regulated through amplifications in 3p12 and 11q22, respectively. These two genes encode proteins that are cofactors of the TEAD family of transcription factors $(30,31)$. However, the exact role of these amplifications in the pathogenesis and progression of DDLS remains to be elucidated.

In summary, we describe the cytogenetic and molecular genetic findings of giant DDLS arising in the thigh of an elderly female. DDLS of the extremity appears to have cytogenetic findings and molecular genetic alterations similar to those detected in retroperitoneal DDLS.

\section{Acknowledgements}

This study was supported in part by the Kaibara Morikazu Medical Science Promotion Foundation, Japan Orthopaedics and Traumatology Foundation, Fukuoka Cancer Society, Clinical Research Foundation, and Medical Care Education Research Foundation. 


\section{References}

1. Dei Tos AP and Pedeutour F: Dedifferentiated liposarcoma In: World Health Organization Classification of Tumours: Pathology and Genetics of Tumours of Soft Tissue and Bone. Fletcher CDM, Unni KK and Mertens F (eds). IARC Press, Lyon, pp38-39, 2002

2. Sandberg AA: Updates on the cytogenetics and molecular genetics of bone and soft tissue tumors: liposarcoma. Cancer Genet Cytogenet 155: 1-24, 2004.

3. Nishio J: Contributions of cytogenetics and molecular cytogenetics to the diagnosis of adipocytic tumors. J Biomed Biotechnol 2011: 524067, 2011.

4. Forus A, Larramendy ML, Meza-Zepeda LA, Bjerkehagen B, Godager LH, Dahlberg AB, Saeter G, Knuutila S and Myklebost O: Dedifferentiation of a well-differentiated liposarcoma to a highly malignant metastatic osteosarcoma: amplification of $12 \mathrm{q} 14$ at al stages and gain of 1q22-q24 associated with metastases. Cancer Genet Cytogenet 125: 100-111, 2001.

5. Horvai AE, DeVries S, Roy R, O'Donnell RJ and Waldman F: Similarity in genetic alterations between paired well-differentiated and dedifferentiated components of dedifferentiated liposarcoma. Mod Pathol 22: 1477-1488, 2009.

6. Tap WD, Eilber FC, Ginther C, Dry SM, Reese N, Barzan-Smith K, Chen HW, Wu H, Eilber FR, Slamon DJ and Anderson L: Evaluation of well-differentiated/de-differentiated liposarcomas by high-resolution oligonucleotide array-based comparative genomic hybridization. Genes Chromosomes Cancer 50: 95-112, 2011.

7. Nishio J, Iwasaki H, Ishiguro M, Ohjimi Y, Fujita C, Ikegami H, Ariyoshi A, Naito M, Kaneko Y and Kikuchi M: Establishment of a novel human dedifferentiated liposarcoma cell line, FU-DDLS-1: conventional and molecular cytogenetic characterization. Int J Oncol 22: 535-542, 2003.

8. Nishio J, Iwasaki H, Ohjimi Y, Ishiguro M, Isayama T, Naito M, Okabayashi H, Kaneko Y and Kikuchi M: Ossifying fibromyxoid tumor of soft parts. Cytogenetic findings. Cancer Genet Cytogenet 133: 124-128, 2002.

9. Shaffer LG, Slovak ML and Campbell LJ (eds): ISCN (2009): An International System for Human Cytogenetic Nomenclature. Karger, Basel, 2009.

10. Nishio J, Iwasaki H, Ohjimi Y, Ishiguro M, Koga T, Isayama T, Naito $\mathrm{M}$ and Kikuchi M: Gain of Xq detected by comparative genomic hybridization in elastofibroma. Int J Mol Med 10: 277-280, 2002

11. Henricks WH, Chu YC, Goldblum JR and Weiss SW: Dedifferentiated liposarcoma: a clinicopathological analysis of 155 cases with a proposal for an expanded definition of dedifferentiation. Am J Surg Pathol 21: 271-281, 1997.

12. Nascimento AG, Kurtin PJ, Guillou L and Fletcher CD: Dedifferentiated liposarcoma: a report of nine cases with a peculiar neurallike whorling pattern associated with metaplastic bone formation. Am J Surg Pathol 22: 945-955, 1998.

13. Fanburg-Smith JC and Miettinen M: Liposarcoma with meningothelial-like whorls: a study of 17 cases of a distinctive histological pattern associated with dedifferentiated liposarcoma. Histopathology 33: 414-424, 1998.

14. Thway K, Robertson D, Thway Y and Fisher C: Dedifferentiated liposarcoma with meningothelial-like whorls, metaplastic bone formation, and CDK4, MDM2, and p16 expression: a morphologic and immunohistochemical study. Am J Surg Pathol 35: 356-363, 2011.

15. Macarenco RS, Erickson-Johnson M, Wang X, Jenkins RB, Nascimento AG and Oliveira AM: Cytogenetic and molecular cytogenetic findings in dedifferentiated liposarcoma with neurallike whorling pattern and metaplastic bone formation. Cancer Genet Cytogenet 172: 147-150, 2007.
16. Italiano A, Bianchini L, Keslair F, Bonnafous S, CardotLeccia N, Coindre JM, Dumollard JM, Hofman P, Leroux A, Mainguené C, Peyrottes I, Ranchere-Vince D, Terrier P, Tran A Gual P and Pedeutour F: HMGA2 is the partner of MDM2 in well-differentiated and dedifferentiated liposarcomas whereas CDK4 belongs to a distinct inconsistent amplicon. Int J Cancer 122: 2233-2241, 2008.

17. Rieker RJ, Weitz J, Lehner B, Egerer G, Mueller A, Kasper B, Schirmacher P, Joos S and Mechtersheimer G: Genomic profiling reveals subsets of dedifferentiated liposarcoma to follow separate molecular pathways. Virchows Arch 456: 277-285, 2010.

18. Conyers R, Young S and Thomas DM: Liposarcoma: molecular genetics and therapeutics. Sarcoma 2011: 483154, 2011.

19. Sirvent N, Coindre JM, Maire G, Hostein I, Keslair F, Guillou L, Ranchere-Vince D, Terrier P and Pedeutour F: Detection of MDM2-CDK4 amplification by fluorescence in situ hybridization in 200 paraffin-embedded tumor samples: utility in diagnosing adipocytic lesions and comparison with immunohistochemistry and real-time PCR. Am J Surg Pathol 31: 1476-1489, 2007.

20. Mariani O, Brennetot C, Coindre JM, Gruel N, Ganem C, Delattre O, Stern MH and Aurias A: JUN oncogene amplification and overexpression block adipocytic differentiation in highly aggressive sarcomas. Cancer Cell 11: 361-374, 2007.

21. Chibon F, Mariani O, Derré J, Mairal A, Coindre JM, Guillou L, Sastre X, Pédeutour F and Aurias A: ASK1 (MAP3K5) as a potential therapeutic target in malignant fibrous histiocytomas with 12q14-q15 and 6q23 amplifications. Genes Chromosomes Cancer 40: 32-37, 2004.

22. Shaulian E and Karin M: AP-1 as a regulator of cell life and death. Nat Cell Biol 4: E131-E136, 2002.

23. Castriota G, Thompson GM, Lin Y, Scherer PE, Moller DE and Berger JP: Peroxisome proliferator-activated receptor gamma agonists inhibit adipocyte expression of alpha1-acid glycoprotein. Cell Biol Int 31: 586-591, 2007.

24. Forus A, Weghuis DO, Smeets D, Fodstad O, Myklebost O and van Kessel AG: Comparative genomic hybridization analysis of human sarcomas: I. Occurrence of genomic imbalances and identification of a novel major amplicon at 1q21-q22 in soft tissue sarcomas. Genes Chromosomes Cancer 14: 8-14, 1995.

25. Szymanska J, Virolainen M, Tarkkanen M, Wiklund T, AskoSeljavaara S, Tukiainen E, Elomaa I, Blomqvist C and Knuutila S: Overrepresentation of 1q21-23 and 12q13-21 in lipoma-like liposarcomas but not in benign lipomas: a comparative genomic hybridization study. Cancer Genet Cytogenet 99: 14-18, 1997.

26. Forus A, Berner JM, Meza-Zepeda LA, Saeter G, Mischke D, Fodstad $\mathrm{O}$ and Myklebost $\mathrm{O}$ : Molecular characterization of a novel amplicon at 1q21-q22 frequently observed in human sarcomas. Br J Cancer 78: 495-503, 1998.

27. Nilsson M, Meza-Zepeda LA, Mertens F, Forus A, Myklebost O and Mandahl N: Amplification of chromosome 1 sequences in lipomatous tumors and other sarcomas. Int J Cancer 109: 363-369, 2004

28. Kresse SH, Berner JM, Meza-Zepeda LA, Gregory SG, Kuo WL, Gray JW, Forus A and Myklebost O: Mapping and characterization of the amplicon near APOA2 in 1q23 in human sarcomas by FISH and array CGH. Mol Cancer 4: 39, 2005.

29. Hélias-Rodzewicz Z, Pérot G, Chibon F, Ferreira C, Lagarde P, Terrier P, Coindre JM and Aurias A: YAP1 and VGLL3, encoding two cofactors of TEAD transcription factors, are amplified and overexpressed in a subset of soft tissue sarcomas. Genes Chromosomes Cancer 49: 1161-1171, 2010.

30. Vaudin P, Delanoue R, Davidson I, Silber J and Zider A: TONDU (TDU), a novel human protein related to the product of vestigial (vg) gene of Drosophila melanogaster interacts with vertebrate TEF factors and substitutes for Vg function in wing formation. Development 126: 4807-4816, 1999.

31. Vassilev A, Kaneko KJ, Shu H, Zhao Y and DePamphilis ML: TEAD/TEF transcription factors utilize the activation domain of YAP65, a Src/Yes-associated protein localized in the cytoplasm. Genes Dev 15: 1229-1241, 2001. 